\title{
Three Nontrivial Solutions for $p$-Laplacian Neumann Problems with a Concave Nonlinearity Near the Origin
}

\author{
Sergiu Aizicovici, Nikolaos S. Papageorgiou, and Vasile Staicu
}

\begin{abstract}
We consider a nonlinear Neumann problem driven by the $p$ Laplacian, with a right-hand side nonlinearity which is concave near the origin. Using variational techniques, combined with the method of upper-lower solutions and with Morse theory, we show that the problem has at least three nontrivial smooth solutions, two of which have a constant sign (one positive and one negative).
\end{abstract}

\section{Introduction}

Let $Z \subseteq \mathbb{R}^{n}$ be a bounded domain with a $C^{2}$ boundary $\partial Z$. In this paper we study the following nonlinear Neumann problem:

$$
\left\{\begin{array}{l}
-\triangle_{p} x(z)+\beta|x(z)|^{p-2} x(z)=f(z, x(z)) \text { a.e. on } Z, \\
\frac{\partial x}{\partial n}=0 \text { on } \partial Z .
\end{array}\right.
$$

Here $\triangle_{p} x(z)=\operatorname{div}\left(\|D x(z)\|_{\mathbb{R}^{N}}^{p-2} D x(z)\right), 2 \leq p<\infty$, is the $p$-Laplacian differential operator, $\beta>0$ and $f(z, x)$ is a Carathéodory nonlinearity. The aim of this work is to prove a three solutions theorem for problem (1.1), when the nonlinearity $f(z,$.$) exhibits a (p-1)$-sublinear behavior near the origin (concave nonlinearity).

Recently, there have been some multiplicity results for Neumann problems driven by the $p$-Laplacian differential operator. We mention the works of Anello [4], Binding-Drabek-Huang [6], Bonanno-Candito [7], Faraci [11], FilippakisGasinski-Papageorgiou [12], Motreanu-Papageorgiou [20], Ricceri [24] and Wu-Tan [28]. In Anello [4], Bonanno-Candito [7], Faraci [11] and Ricceri [24], the authors consider nonlinear eigenvalue problems and prove the existence of multiple solutions when the nonlinearity is oscillating and the parameter belongs to an open interval in $\mathbb{R}_{+}$. In these works, the key assumption is that $p>N$ (low dimensional problem), which implies that the Sobolev space $W^{1, p}(Z)$ is embedded

2000 Mathematics Subject Classification. 35J25, 35J70, 58E05.

Key words and phrases. p-Laplacian, concave nonlinearity, critical groups, Poincaré-Hopf formula, local minimizer.

The third author acknowledges partial financial support from the Portuguese Foundation for Sciences and Technology (FCT) under the project POCI/MAT/55524/2004. 
compactly in $C(\bar{Z})$. The approach in all these papers is essentially similar, and is based on an abstract variational principle due to Ricceri [23]. In Wu-Tan [28], it is again assumed that $p>N$ and the approach (which is variational) is based on the critical point theory. Binding-Drabek-Huang [6] considered problems with a particular right-hand side nonlinearity, of the form $\lambda a(z)|x|^{p-2} x+b(z)|x|^{q-2} x$, with $a, b \in L^{\infty}(Z), \lambda \in \mathbb{R}, 1<p<N$ and $1<q<p^{*}$, where $p^{*}$ is the critical Sobolev exponent given by

$$
p^{*}= \begin{cases}\frac{N p}{N-p} & \text { if } p<N \\ +\infty & \text { if } p \geq N .\end{cases}
$$

They prove the existence of one or two positive solutions.

Finally, we should also mention the recent work [1], which is concerned with problem (1.1) with a $p$-superlinear potential $F(z, x)=\int_{0}^{x} f(s, x) d s$ (where $f(z,$. satisfies the Ambrosetti-Rabinowitz condition). The authors prove multiplicity theorems, providing precise information about the sign of the solutions.

None of the aforementioned works treats nonlinearities which are concave near the origin. Problems with concave nonlinearities were considered in the context of semilinear problems (i.e., $p=2$ ) or Dirichlet problems, by de Paiva-Massa [10], Li-Wu-Zhou [16], Perera [21] and Wu-Yang [27]. For Dirichlet problems with the $p$-Laplacian, we mention the work of Garcia Azorero-Manfredi-Peral Alonso [13], where a nonlinear eigenvalue problem is considered, with a nonlinearity of the form $\lambda|x|^{r-2} x+|x|^{q-2} x$, with $\lambda>0$ and $1<r<p<q<p^{*}$ (concaveconvex nonlinearity). Their work extended earlier results for the semilinear case by Ambrosetti-Brezis-Cerami [3].

Our approach here is different from all of the above works. It combines variational techniques with the method of upper-lower solutions and with Morse theory (in particular, critical groups).

The rest of the paper is organized as follows. In Section 2 we present some background material and some general auxiliary results, which we will need in the sequel. In Section 3, employing variational arguments in combination with the method of upper-lower solutions, we produce two nontrivial smooth solutions of constant sign (one positive and the other negative). Finally, in Section 4, using suitable tools from Morse theory, we establish the existence of a third nontrivial smooth solution.

\section{Background material}

In the analysis of problem (1.1) we will use the following two spaces:

$W_{n}^{1, p}(Z)=\left\{x \in W^{1, p}(Z): x_{k} \rightarrow x\right.$ in $W^{1, p}(Z), x_{k} \in C^{\infty}(\bar{Z}), \frac{\partial x_{k}}{\partial n}=0$ on $\left.\partial Z\right\}$ and

$$
C_{n}^{1}(\bar{Z})=\left\{x \in C^{1}(\bar{Z}): \frac{\partial x}{\partial n}=0 \text { on } \partial Z\right\}
$$

where by $\bar{Z}$ we denote the closure of the domain $Z$. Both are ordered Banach spaces, with order cones given by

$$
W_{+}=\left\{x \in W_{n}^{1, p}(Z): x(z) \geq 0 \text { a.e. on } Z\right\}
$$


and, respectively,

$$
C_{+}=\left\{x \in C_{n}^{1}(\bar{Z}): x(z) \geq 0 \text { for all } z \in \bar{Z}\right\} .
$$

We know that int $C_{+} \neq \varnothing$ (where int stands for the interior), with

$$
\operatorname{int} C_{+}=\left\{x \in C_{+}: x(z)>0 \text { for all } z \in \bar{Z}\right\} .
$$

In what follows, by $\|\cdot\|_{p}$ we denote the norm of $L^{p}(Z)$ (or $L^{p}\left(Z, \mathbb{R}^{N}\right)$ ), and by $\|\cdot\|$ the norm of $W^{1, p}(Z)$. The norm of $W_{n}^{1, p}(Z)$ is also denoted by $\|$.$\| .$

The next result, (see, e.g., [1]), compares $C_{n}^{1}(\bar{Z})$ and $W_{n}^{1, p}(Z)$-local minimizers for a large class of energy functionals. It extends to earlier results of Neumann problems by Brezis-Nirenberg [8] (for $p=2$ ) and by Garcia Azorero-Manfredi-Peral Alonso [13] (for $p \neq 2$ ), which were concerned with Dirichlet boundary conditions.

So, consider a nonlinearity $\widehat{f}: Z \times \mathbb{R} \rightarrow \mathbb{R}$ satisfying the following hypotheses:

$\left(H_{0}\right) \quad$ (i) for all $x \in \mathbb{R}, z \rightarrow \widehat{f}(z, x)$ is measurable;

(ii) for almost all $z \in Z, x \rightarrow \widehat{f}(z, x)$ is continuous;

(iii) for almost all $z \in Z$ and all $x \in \mathbb{R}$,

$$
|\widehat{f}(z, x)| \leq \widehat{a}(z)+\widehat{c}|x|^{r-1}
$$

where $\widehat{a} \in L^{\infty}(Z)_{+}, \widehat{c}>0$ and $1<r<p^{*}$, with $p^{*}$ defined by (1.2).

Let $\widehat{F}(z, x)=\int_{0}^{x} \widehat{f}(z, s) d s$ and consider the functional $\widehat{\varphi}: W_{n}^{1, p}(Z) \rightarrow \mathbb{R}$ defined by

$$
\widehat{\varphi}(x)=\frac{1}{p}\|D x\|_{p}^{p}-\int_{Z} \widehat{F}(z, x(z)) d z \text { for all } x \in W_{n}^{1, p}(Z) .
$$

Evidently $\widehat{\varphi} \in C^{1}\left(W_{n}^{1, p}(Z)\right)$.

Proposition 1. Let $\left(H_{0}\right)$ be satisfied. If $x_{0} \in W_{n}^{1, p}(Z)$ is a local $C_{n}^{1}(\bar{Z})$ minimizer of $\widehat{\varphi}$, i.e., there exists $\rho_{1}>0$ such that

$$
\widehat{\varphi}\left(x_{0}\right) \leq \widehat{\varphi}\left(x_{0}+h\right) \text { for all } h \in C_{n}^{1}(\bar{Z}),\|h\|_{C_{n}^{1}(\bar{Z})} \leq \rho_{1},
$$

then $x_{0} \in C_{n}^{1}(\bar{Z})$ and it is a local $W_{n}^{1, p}(Z)$-minimizer of $\widehat{\varphi}$, i.e., there exists $\rho_{2}>0$ such that

$$
\widehat{\varphi}\left(x_{0}\right) \leq \widehat{\varphi}\left(x_{0}+h\right) \text { for all } h \in W_{n}^{1, p}(Z),\|h\| \leq \rho_{2} .
$$

Next let us recall the notions of upper and lower solutions for problem (1.1).

Definition 1. (a) An upper solution for problem (1.1) is a function $\bar{x} \in C^{1}(\bar{Z})$ such that

$$
\frac{\partial \bar{x}}{\partial n} \geq 0 \text { on } \partial Z
$$

and

$$
\int_{Z}\|D \bar{x}\|_{\mathbb{R}^{N}}^{p-2}(D \bar{x}, D h)_{\mathbb{R}^{N}} d z+\beta \int_{Z}|\bar{x}|^{p-2} \bar{x} h d z \geq \int_{Z} f(z, \bar{x}) h d z
$$

for all $h \in W_{+}$. We say that $\bar{x}$ is a strict upper solution for problem (1.1), if it is an upper solution but it is not a solution of (1.1).

(b) A lower solution for problem (1.1) is a function $\underline{x} \in C^{1}(\bar{Z})$ such that

$$
\frac{\partial \underline{x}}{\partial n} \leq 0 \text { on } \partial Z
$$


and

$$
\int_{Z}\|D \underline{x}\|_{\mathbb{R}^{N}}^{p-2}(D \underline{x}, D h)_{\mathbb{R}^{N}} d z+\beta \int_{Z}|\underline{x}|^{p-2} \underline{x} h d z \leq \int_{Z} f(z, \underline{x}) h d z
$$

for all $h \in W_{+}$. We say that $\underline{x}$ is a strict lower solution, if it is a lower solution but it is not a solution of (1.1).

Now, let us recall some basic notions and results from Morse theory, which we will need to produce the third nontrivial smooth solution for problem (1.1).

Let $X$ be a Banach space and $\varphi \in C^{1}(X)$. For every $c \in \mathbb{R}$, we set

$$
\begin{gathered}
\varphi^{c}=\{x \in X: \varphi(x) \leq c\},(\text { the sublevel set of } \varphi \text { at } \mathrm{c}), \\
K=\left\{x \in X: \varphi^{\prime}(x)=0\right\},(\text { the critical set of } \varphi),
\end{gathered}
$$

and

$$
\left.K_{c}=\{x \in K: \varphi(x)=c\} \text { (the critical set of } \varphi \text { at level } \mathrm{c} \in \mathbb{R}\right) .
$$

Let $Y$ be a subspace of a Hausdorff topological space $V$ and let $n \geq 0$ be an integer. By $H_{n}(V, Y)$ we denote the $n^{t h}$-singular homology group of the pair $(V, Y)$ with integer coefficients. If $x_{0} \in X$ is an isolated critical point of $\varphi$ with $\varphi\left(x_{0}\right)=c$, then the critical groups of $\varphi$ at $x_{0}$ are defined by

$$
C_{n}\left(\varphi, x_{0}\right)=H_{n}\left(\varphi^{c} \cap U,\left(\varphi^{c} \cap U\right) \backslash\left\{x_{0}\right\}\right), n \geq 0,
$$

where $U$ is a neighborhood of $x_{0}$ such that $K \cap \varphi^{c} \cap U=\left\{x_{0}\right\}$. By the excision property of the singular homology theory, we infer that the above definition of critical groups is independent of $U$ (see Chang [9], and Mawhin-Willem [18]).

In what follows, we assume that $\varphi$ satisfies the usual $P S$-condition. Namely, if $\left\{x_{n}\right\}_{n \in \mathbb{N}} \subseteq X$ is a sequence such that $\left|\varphi\left(x_{n}\right)\right| \leq M$ for some $M>0$ and all $n \geq 1$, and $\varphi^{\prime}\left(x_{n}\right) \rightarrow 0$ in $X^{*}$, then $\left\{x_{n}\right\}_{n \in \mathbb{N}}$ has a strongly convergent subsequence (see [9, p. 20], [14, p. 611], and [18, p. 81]).

Assume that $-\infty<\inf \varphi(K)$ and let $c<\inf \varphi(K)$. Then, the critical groups of $\varphi$ at infinity are defined by

$$
C_{n}(\varphi, \infty)=H_{n}\left(H, \varphi^{c}\right) \text { for all } n \geq 0,
$$

(see Bartsch-Li [5]). The deformation lemma (see, for example, [9, p. 21]) implies that this definition is independent of the choice of $c$. If $\varphi \in C^{1}(X)$ and $K=\left\{x_{0}\right\}$, then Morse theory implies that

$$
C_{n}\left(\varphi, x_{0}\right)=C_{n}(\varphi, \infty) \text { for all } n \geq 0 .
$$

In particular, if $x_{0}$ is an isolated critical point of $\varphi$ and $C_{n}\left(\varphi, x_{0}\right) \neq C_{n}(\varphi, \infty)$ for some $n \geq 0$, then $\varphi$ must have another critical point, distinct from $x_{0}$. Moreover, if $K$ is finite, then the Morse type numbers of $\varphi$ are defined by

$$
M_{n}=\sum_{x \in K} \operatorname{rank} C_{n}(\varphi, x), n \geq 0,
$$

and the Betti-type numbers of $\varphi$, are defined by

$$
\beta_{n}=\operatorname{rank} C_{n}(\varphi, \infty), n \geq 0 .
$$

By Morse theory (see Bartsch-Li [5], Chang [9], and Mawhin-Willem [18]), we have the Poincaré-Hopf formula

$$
\sum_{n \geq 0}(-1)^{n} M_{n}=\sum_{n \geq 0}(-1)^{n} \beta_{n}
$$


The next result is useful in the computation of critical groups at infinity. It is related to Lemma 2.4 of Perera-Schechter [22], where $X$ is a Hilbert space.

Proposition 2. Let $(X,\|\cdot\|)$ be a Banach space of dual $\left(X^{*},\|\cdot\|_{*}\right)$ and let $(t, x) \rightarrow \varphi_{t}(x)$ be a function in $C^{1}([0,1] \times X)$, such that $x \rightarrow \varphi_{t}^{\prime}(x)$ and $x \rightarrow$ $\partial_{t} \varphi_{t}(x)$ are both locally Lipschitz. (Here by $\varphi_{t}^{\prime}(x)$ we denote the Frechet derivative of $x \rightarrow \varphi_{t}(x)$ and by $\partial_{t} \varphi_{t}$ the derivative of $t \rightarrow \varphi_{t}(x)$.) If we can find $R>0$ such that

$$
\inf \left\{\left\|\varphi_{t}^{\prime}(x)\right\|_{*}: t \in[0,1],\|x\|>R\right\}>0
$$

and

$$
\xi_{R}:=\inf \left\{\varphi_{t}(x): t \in[0,1],\|x\| \leq R\right\}>-\infty
$$

then for all $c<\xi_{R}$, the set $\varphi_{0}^{c}$ is homemorphic to a subset of $\varphi_{1}^{c}$.

Proof. Note that by virtue of $(2.2)$, for every $t \in[0,1]$, we have

$$
K_{t}=\left\{x \in X: \varphi_{t}^{\prime}(x)=0\right\} \subseteq \bar{B}_{R}
$$

with $\bar{B}_{R}=\{x \in X:\|x\| \leq R\}$. Because of (2.4) and since by hypothesis $\varphi \in$ $C^{1}([0,1] \times X)$, it follows (see, for example, $[\mathbf{1 8}$, p. 127]) that there exists a pseudogradient vector field $\widehat{v}=\left(v_{0}, v\right):[0,1] \times\left(X \backslash \bar{B}_{R}\right) \rightarrow[0,1] \times X$ corresponding to $\varphi$. Recalling the construction of the pseudogradient vector field in Chang [9, p. 19], we see that we can take $v_{0}(t, x)=\partial_{t} \varphi_{t}(x)$. By definition, the map $(t, x) \rightarrow v_{t}(x)$ is locally Lipschitz and in fact, for every $t \in[0,1], v_{t}$ is a pseudogradient vector field corresponding to the function $\varphi_{t}$ (see Chang [9, p. 19]). Hence, for every $(t, x) \in[0,1] \times\left(X \backslash \bar{B}_{R}\right)$, we have

$$
\left\langle\varphi_{t}^{\prime}(x), v_{t}(x)\right\rangle \geq\left\|\varphi_{t}^{\prime}(x)\right\|_{*}^{2}
$$

where by $\langle.,$.$\rangle we denote the duality brackets for the pair \left(X^{*}, X\right)$. The map $w:[0,1] \times\left(X \backslash \bar{B}_{R}\right) \rightarrow X$ given by

$$
w_{t}(x)=-\frac{\left|\partial_{t} \varphi_{t}(x)\right|}{\left\|\varphi_{t}^{\prime}(x)\right\|_{*}^{2}} v_{t}(x),
$$

is well-defined and locally Lipschitz. Because of (2.3), we can fix $c \in \mathbb{R}$,

$$
c<\inf \left\{\varphi_{t}(x): t \in[0,1],\|x\| \leq R\right\}
$$

such that $\varphi_{0}^{c} \neq \varnothing$ or $\varphi_{1}^{c} \neq \varnothing$. (If no such $c$ can be found, then

$$
C_{n}\left(\varphi_{0}, \infty\right)=C_{n}\left(\varphi_{1}, \infty\right)=\delta_{n, 0} \mathbb{Z}
$$

and so we are done). Without any loss of generality, we may assume that $\varphi_{0}^{c} \neq \varnothing$ (the argument is similar if $\varphi_{1}^{c} \neq \varnothing$ ). Let $y \in \varphi_{0}^{c}$ and consider the Cauchy problem

$$
\frac{d}{d t} \eta(t)=w_{t}(\eta(t)) \text { for all } t \in[0,1], \eta(0)=y
$$


From the local existence theorem (see Gasinski-Papageorgiou [14, p. 618]), we know that $(2.8)$, admits a local flow $\eta(t)$. On account of $(2.5),(2.6)$ and $(2.8)$, we have

$$
\begin{aligned}
\frac{d}{d t} \varphi_{t}(\eta(t)) & =\left\langle\varphi_{t}^{\prime}(\eta(t)), \frac{d}{d t} \eta(t)\right\rangle+\partial_{t} \varphi_{t}(\eta(t)) \\
& =\left\langle\varphi_{t}^{\prime}(\eta(t)), w_{t}(\eta(t))\right\rangle+\partial_{t} \varphi_{t}(\eta(t)) \\
& \leq-\left|\partial_{t} \varphi_{t}(\eta(t))\right|+\partial_{t} \varphi_{t}(\eta(t)) \\
& \leq 0
\end{aligned}
$$

Therefore, $t \rightarrow \varphi_{t}(\eta(t))$ is decreasing and so we have $\varphi_{t}(\eta(t)) \leq \varphi_{0}(\eta(0))=$ $\varphi_{0}(y) \leq c$ (recall that $\left.y \in \varphi_{0}^{c}\right)$. Because of $(2.7)$, we have that $\|\eta(t)\|>R$. Consequently, $\varphi_{t}^{\prime}(\eta(t)) \neq 0$ and so the flow $\eta$ is in fact global. Moreover, it can be reversed by replacing $\varphi_{t}$ by $\varphi_{1-t}$. Therefore, $\eta(1)$ is a homeomorphism between $\varphi_{0}^{c}$ and a subset of $\varphi_{1}^{c}$.

Let us recall the following notion from nonlinear operator theory. Let $X$ be a Banach space, $X^{*}$ its topological dual and as before, let $\langle.,$.$\rangle denote the duality$ brackets for the pair $\left(X^{*}, X\right)$.

Definition 2. A map $A: X \rightarrow X^{*}$ is said to be of type $(S)_{+}$, if for every sequence $\left\{x_{n}\right\}_{n \geq 1} \subseteq X$ such that $x_{n} \stackrel{w}{\longrightarrow} x$ in $X$ and

$$
\limsup _{n \rightarrow \infty}\left\langle A\left(x_{n}\right), x_{n}-x\right\rangle \leq 0,
$$

one has

$$
x_{n} \rightarrow x \text { in } X \text {. }
$$

(Here and in the sequel, we use “ $\stackrel{w}{\longrightarrow}$ to denote weak convergence).

Let $X=W_{n}^{1, p}(Z), X^{*}=W_{n}^{1, p}(Z)^{*}$ and consider the nonlinear operator $A: W_{n}^{1, p}(Z) \rightarrow W_{n}^{1, p}(Z)^{*}$ defined by

$$
\langle A(x), y\rangle=\int_{Z}\|D x\|_{\mathbb{R}^{N}}^{p-2}(D x, D y)_{\mathbb{R}^{N}} d z \text { for all } x, y \in W_{n}^{1, p}(Z) .
$$

The following result is well-known; see, e.g., $[\mathbf{1}]$.

Proposition 3. The nonlinear operator $A: W_{n}^{1, p}(Z) \rightarrow W_{n}^{1, p}(Z)^{*}$ defined by (2.9) is bounded, continuous, monotone and of type $(S)_{+}$.

REMARK 1. In particular, $A$ is maximal monotone and so, pseudomonotone, as well (see Gasinski-Papageorgiou [14, p. 334]).

\section{Solutions of constant sign}

In this section, using variational techniques together with the method of upperlower solutions, we produce two nontrivial smooth solutions of constant sign, one positive and the other negative. For this, we do not need the restriction $p \geq 2$. So, in this section, $1<p<\infty$.

The hypotheses on the nonlinearity $f(z, x)$ are the following:

$\mathbf{H}(f): f: Z \times \mathbb{R} \rightarrow \mathbb{R}$ is a function such that $f(z, 0)=0$ a.e. on $Z$ and

(i) for all $x \in \mathbb{R}, z \rightarrow f(z, x)$ is measurable;

(ii) for almost all $z \in Z, x \rightarrow f(z, x)$ is continuous; 
(iii) for every $\rho>0$, there exists $a_{\rho} \in L^{\infty}(Z)_{+}$such that

$$
|f(z, x)| \leq a_{\rho}(z) \text { for a.a. } z \in Z \text { and all }|x| \leq \rho
$$

$(i v)$ there exists $\theta \in L^{\infty}(Z)_{+}$such that $\theta(z) \leq \beta$ a.e. on $Z$, with strict inequality on a set of positive measure, and if $F(z, x)=\int_{0}^{x} f(z, s) d s$, then

$$
\limsup _{|x| \rightarrow \infty} \frac{p F(z, x)}{|x|^{p}} \leq \theta(z) \text { uniformly for a.a. } z \in Z ;
$$

$(v)$ there exist $\delta>0, r \in(1, p)$ and $c_{0}>0$ such that

$$
c_{0}|x|^{r} \leq F(z, x) \text { for a.a. } z \in Z \text { and all }|x| \leq \delta \text {; }
$$

(vi) for almost all $z \in Z$, we have

$$
f(z, x) x \geq 0 \text { for all } x \in \mathbb{R} \quad \text { (sign condition) }
$$

and

$$
p F(z, x)-f(z, x) x>0 \text { for all } x \neq 0 .
$$

REMARK 2. Hypothesis $\mathbf{H}(f)(v)$ implies that the nonlinearity $f(z,$.$) exhibits$ an $(r-1)$-sublinear growth near the origin (concave nonlinearity near the origin). For example, the nonlinearity

$$
f(x, x)=\theta(z)|x|^{p-2} x+|x|^{r-2} x
$$

with $1<r<p$ and $\theta \in L^{\infty}(Z)_{+}$as in assumption $\mathbf{H}(f)(i v)$ satisfies hypotheses $\mathbf{H}(f)$.

First, we will produce a strict upper solution of (1.1). By virtue of hypotheses $\mathbf{H}(f)(i i i),(i v)$ and $(v i)$, given $\varepsilon>0$, we can find $\xi_{\varepsilon} \in L^{\infty}(Z)_{+}, \xi_{\varepsilon} \neq 0$ and $\eta_{\varepsilon}>0$ such that

$$
(\theta(z)+\varepsilon) x^{p-1}+\xi_{\varepsilon}(z)-f(z, x) \geq \eta_{\varepsilon}>0 \text { for a.a. } z \in Z \text { and all } x \geq 0 .
$$

To produce a strict upper solution for problem (1.1), we will need the following lemma, which underlines the significance of the nonuniform resonance hypothesis $\mathbf{H}(f)(i v)$.

LEMMA 1. If $\theta \in L^{\infty}(Z)_{+}, \theta(z) \leq \beta$ a.e. on $Z$, with strict inequality on a set of positive measure, then there exists $\widehat{\xi}_{0}>0$ such that

$$
\psi(x)=\|D x\|_{p}^{p}+\beta\|x\|_{p}^{p}-\int_{Z} \theta(z)|x(z)|^{p} d z \geq \widehat{\xi}_{0}\|x\|^{p} \quad \text { for all } x \in W^{1, p}(Z) .
$$

Proof. Note that $\psi \geq 0$. We argue by contradiction. So, suppose that the lemma is not true. Exploiting the $p$-homogeneity of $\psi$, we can find a sequence $\left\{x_{n}\right\}_{n \in \mathbb{N}} \subseteq W^{1, p}(Z)$ such that

$$
\left\|x_{n}\right\|=1 \text { and } \psi\left(x_{n}\right) \downarrow 0 .
$$

By passing to a suitable subsequence we may assume that

$$
x_{n} \stackrel{w}{\longrightarrow} x \text { in } W^{1, p}(Z) \text { and } x_{n} \rightarrow x \text { in } L^{p}(Z) .
$$

Then we have

$$
\|D x\|_{p}^{p} \leq \liminf _{n \rightarrow \infty}\left\|D x_{n}\right\|_{p}^{p}, \beta\left\|x_{n}\right\|_{p}^{p} \rightarrow \beta\|x\|_{p}^{p}
$$


and

So, in the limit as $n \rightarrow \infty$, we obtain

$$
\int_{Z} \theta(z)\left|x_{n}(z)\right|^{p} d z \rightarrow \int_{Z} \theta(z)|x(z)|^{p} d z .
$$

$$
\|D x\|_{p}^{p}+\beta\|x\|_{p}^{p} \leq \int_{Z} \theta(z)|x(z)|^{p} d z .
$$

Hence

$$
\|D x\|_{p}^{p} \leq \int_{Z}(\theta(z)-\beta)|x(z)|^{p} d z \leq 0
$$

therefore

$$
x \equiv c \in \mathbb{R} .
$$

If $c=0$, then $\left\|D x_{n}\right\|_{p} \rightarrow 0$ and so $x_{n} \rightarrow 0$ in $W^{1, p}(Z)$, a contradiction to the fact that $\left\|x_{n}\right\|=1$ for all $n \geq 1$. So, $c \neq 0$. From (3.2), we have

$$
0 \leq|c|^{p} \int_{Z}(\theta(z)-\beta) d z<0,
$$

again a contradiction. This proves the lemma.

Proposition 4. If hypotheses $\mathbf{H}(f)$ hold, then problem (1.1) admits a strict upper solution $\bar{x} \in \operatorname{int} C_{+}$.

Proof. Consider the nonlinear operator $\widehat{K}_{p}: L^{p}(Z) \rightarrow L^{p^{\prime}}(Z)\left(\frac{1}{p}+\frac{1}{p^{\prime}}=1\right)$ defined by

$$
\widehat{K}_{p}(x)(.)=|x(.)|^{p-2} x(.) \text { for all } x \in L^{p}(Z) .
$$

Clearly $\widehat{K}_{p}$ is continuous and bounded (i.e., it maps bounded sets to bounded ones). Moreover, by virtue of the compact embedding of $W^{1, p}(Z)$ into $L^{p}(Z)$, it follows that

$$
K_{p}=\left.\widehat{K}_{p}\right|_{W^{1, p}(Z)}: W^{1, p}(Z) \rightarrow W^{1, p}(Z)^{*}
$$

is completely continuous (i.e., it is sequentially weakly-strongly continuous). Therefore, by Remark 1, the map $V: W^{1, p}(Z) \rightarrow W^{1, p}(Z)^{*}$ defined by

$$
V(x)=A(x)+\beta K_{p}(x)-(\theta(.)+\varepsilon) K_{p}(x)
$$

is pseudomonotone. Also, for every $x \in W^{1, p}(Z)$, we have

$$
\langle V(x), x\rangle=\|D x\|_{p}^{p}+(\beta-\varepsilon)\|x\|_{p}^{p}-\int_{Z} \theta(z)|x(z)|^{p} d z \geq\left(\widehat{\xi}_{0}-\varepsilon\right)\|x\|^{p}
$$

(see Lemma 1). Choosing $0<\varepsilon<\widehat{\xi}_{0}$, from(3.3) we infer that $V$ is coercive. But a pseudomonotone coercive operator is surjective (see Gasinski-Papageorgiou [14, p. 336]). Therefore, we can find $\bar{x} \in W^{1, p}(Z)$ such that

$$
V(\bar{x})=A(\bar{x})+\beta K_{p}(\bar{x})-(\theta+\varepsilon) K_{p}(\bar{x})=\xi_{\varepsilon},
$$

where $\xi_{\varepsilon}$ is as in (3.1). Since $\xi_{\varepsilon} \neq 0,(3.4)$ implies that $\bar{x} \neq 0$. Recall that

$$
\bar{x}=\bar{x}^{+}-\bar{x}^{-}, \text {with } \bar{x}^{+}=\max \{\bar{x}, 0\} \text { and } \bar{x}^{-}=-\min \{\bar{x}, 0\} .
$$

On (3.4) we act with the test function $-\bar{x}^{-} \in W_{n}^{1, p}(Z)$ and we obtain

$$
\left\|D \bar{x}^{-}\right\|_{p}^{p}+\beta\left\|\bar{x}^{-}\right\|_{p}^{p}-\int_{Z} \theta(z)\left|\bar{x}^{-}(z)\right|^{p} d z-\varepsilon\left\|\bar{x}^{-}\right\|^{p} \leq 0
$$


hence

$$
\left(\widehat{\xi}_{0}-\varepsilon\right)\left\|\bar{x}^{-}\right\|^{p} \leq 0
$$

(see Lemma 1). Inasmuch as $\varepsilon<\widehat{\xi}_{0}$, from (3.5) it follows that $\bar{x}^{-}=0$, hence $\bar{x} \geq 0, \bar{x} \neq 0$. On account of (3.4) and the nonlinear Green identity (cf. MotreanuPapageorgiou $[\mathbf{2 0}])$, we get

$$
\left\{\begin{array}{l}
-\triangle_{p} \bar{x}(z)+\beta \bar{x}(z)^{p-1}=(\theta(z)+\varepsilon) \bar{x}(z)^{p-1}+\xi_{\varepsilon}(z) \text { a.e. on } Z \\
\frac{\partial \bar{x}}{\partial n}=0 \text { on } \partial Z .
\end{array}\right.
$$

From (3.6) and Theorem 7.1, p. 286 of Ladyzhenskaya-Uraltseva [15], we deduce that $\bar{x} \in L^{\infty}(Z)$. Then, invoking Theorem 2 of Lieberman [17], we infer that $\bar{x} \in C_{+}$.

Note that (3.6) implies

$$
\triangle_{p} \bar{x}(z) \leq \beta \bar{x}(z)^{p-1} \text { a.e. on } Z \text {. }
$$

Hence, by virtue of the nonlinear strong maximum principle of Vazquez [25], we obtain $\bar{x}(z)>0$ for all $z \in Z$. Suppose that for some $z_{0} \in \partial Z$, we have $\bar{x}\left(z_{0}\right)=0$. Then, from Vazquez [25] (Theorem 5), it follows that

$$
\frac{\partial \bar{x}}{\partial n}\left(z_{0}\right)<0
$$

which contradicts (3.6). This proves that $\bar{x}(z)>0$ for all $z \in \bar{Z}$, i.e., $\bar{x} \in \operatorname{int} C_{+}$. Because of (3.1), we see that $\bar{x} \in \operatorname{int} C_{+}$is a strict upper solution for problem (1.1) in the sense of Definition 1(a).

Let $g \in L^{\infty}(Z)$ and consider the following Neumann problem

$$
\left\{\begin{array}{l}
-\triangle_{p} x(z)+\beta|x(z)|^{p-2} x(z)=g(z) \text { a.e. on } Z, \\
\frac{\partial x}{\partial n}=0 \text { on } \partial Z .
\end{array}\right.
$$

From the maximal monotonicity and coercivity of the operator $x \rightarrow A(x)+\beta K_{p}(x)$, we infer that the problem (3.7) has a solution $S(g) \in W_{n}^{1, p}(Z)$, which is unique due to the strict monotonicity of the operator. Moreover, the nonlinear regularity theory implies that $S(g) \in C_{n}^{1}(\bar{Z})$. We examine the monotonicity properties of the map $g \rightarrow S(g)$.

Lemma 2. The map $S: L^{\infty}(Z) \rightarrow C^{1}(\bar{Z})$ is increasing, i.e., if $g_{1} \leq g_{2}$ in $L^{\infty}(Z)$, then $S\left(g_{1}\right) \leq S\left(g_{2}\right)$ in $C^{1}(\bar{Z})$.

Proof. Suppose that $g_{1}, g_{2} \in L^{\infty}(Z)$ and assume that $g_{1} \leq g_{2}$ in $L^{\infty}(Z)$ (i.e., $g_{1}(z) \leq g_{2}(z)$ a.e. on $\left.Z\right)$. Set $x_{1}=S\left(g_{1}\right), x_{2}=S\left(g_{2}\right)$. Then

$$
A\left(x_{1}\right)+\beta K_{p}\left(x_{1}\right)=g_{1} \text { and } A\left(x_{2}\right)+\beta K_{p}\left(x_{2}\right)=g_{2} \text {. }
$$

We have

$$
\begin{aligned}
\left\langle A\left(x_{2}\right)-A\left(x_{1}\right),\left(x_{1}-x_{2}\right)^{+}\right\rangle & +\beta \int_{\left\{x_{1}>x_{2}\right\}}\left(\left|x_{2}\right|^{p-2} x_{2}-\left|x_{1}\right|^{p-2} x_{1}\right)\left(x_{1}-x_{2}\right) d z \\
& =\int_{Z}\left(g_{2}-g_{1}\right)\left(x_{1}-x_{2}\right)^{+} d z \\
& \geq 0
\end{aligned}
$$


hence

$$
\begin{aligned}
\int_{\left\{x_{1}>x_{2}\right\}} & \left(\left\|D x_{2}\right\|_{\mathbb{R}^{N}}^{p-2} D x_{2}-\left\|D x_{1}\right\|_{\mathbb{R}^{N}}^{p-2} D x_{1}, D x_{1}-D x_{2}\right)_{\mathbb{R}^{n}} d z \\
& +\beta \int_{\left\{x_{1}>x_{2}\right\}}\left(\left|x_{2}\right|^{p-2} x_{2}-\left|x_{1}\right|^{p-2} x_{1}\right)\left(x_{1}-x_{2}\right) d z \\
& \geq 0 .
\end{aligned}
$$

But, due to the strict monotonicity of the map $\mathbb{R}^{N} \ni \xi \rightarrow\|\xi\|_{\mathbb{R}^{N}}^{p-2} \xi$ and $\mathbb{R} \ni y \rightarrow$ $|y|^{p-2} y$, the left hand side of (3.8) is strictly negative, a contradiction unless

$$
\left|\left\{x_{1}>x_{2}\right\}\right|_{N}=0
$$

where by $|\cdot|_{N}$ we denote the Lebesgue measure on $\mathbb{R}^{N}$. Hence $x_{1} \leq x_{2}$.

Note that $x \equiv 0$ is a solution of the problem (1.1). We truncate the nonlinearity $f(z, x)$ at the pair $\{0, \bar{x}\}$, namely, we introduce

$$
\widehat{f}_{+}(z, x)= \begin{cases}0 & \text { if } x \leq 0 \\ f(z, x) & \text { if } 0 \leq x \leq \bar{x}(z) \\ f(z, \bar{x}(z)) & \text { if } \bar{x}(z) \leq x\end{cases}
$$

Evidently, $\widehat{f}_{+}(z, x)$ is a Carathédory function, i.e., for all $x \in \mathbb{R}, z \rightarrow \widehat{f}_{+}(z, x)$ is measurable and for almost all $z \in Z, x \rightarrow \widehat{f}_{+}(z, x)$ is continuous. We set

$$
\widehat{F}_{+}(z, x)=\int_{0}^{x} \widehat{f}_{+}(z, s) d s
$$

(the primitive of $\widehat{f}_{+}(z,$.$) ) and consider the functional \widehat{\varphi}_{+}: W_{n}^{1, p}(Z) \rightarrow \mathbb{R}$ defined by

$$
\widehat{\varphi}_{+}(x)=\frac{1}{p}\|D x\|_{p}^{p}+\frac{\beta}{p}\|x\|_{p}^{p}-\int_{Z} \widehat{F}_{+}(z, x(z)) d z \text { for all } x \in W_{n}^{1, p}(Z) .
$$

We also consider $\varphi: W_{n}^{1, p}(Z) \rightarrow \mathbb{R}$, the Euler functional for the problem (1.1), defined by

$$
\varphi(x)=\frac{1}{p}\|D x\|_{p}^{p}+\frac{\beta}{p}\|x\|_{p}^{p}-\int_{Z} F(z, x(z)) d z \text { for all } x \in W_{n}^{1, p}(Z) .
$$

Clearly, $\widehat{\varphi}_{+}, \varphi \in C^{1}\left(W_{n}^{1, p}(Z)\right)$.

Proposition 5. If hypotheses $\mathbf{H}(f)$ hold, then problem (1.1) admits a solution $x_{0} \in \operatorname{int} C_{+}$, which is a local minimizer of $\varphi$.

Proof. Exploiting the compact embedding of $W_{n}^{1, p}(Z)$ into $L^{p}(Z)$, we can easily check that $\widehat{\varphi}_{+}$is sequentially weakly lower semicontinuous. Moreover, note that we can find $M_{1}>0$ such that

$$
\left|\int_{Z} \widehat{F}_{+}(z, x(z)) d z\right| \leq M_{1} \text { for all } x \in W_{n}^{1, p}(Z) .
$$

Hence $\widehat{\varphi}_{+}$is coercive. Invoking the theorem of Weierstrass (see [14, p. 711]), we can find $x_{0} \in W_{n}^{1, p}(Z)$ such that

$$
\widehat{\varphi}_{+}\left(x_{0}\right)=\inf \left\{\widehat{\varphi}_{+}(x): x \in W_{n}^{1, p}(Z)\right\} \text {. }
$$


We claim that $x_{0} \neq 0$. To this end, let $\delta>0$ be as in hypothesis $\mathbf{H}(f)(v)$ and let $c \in(0, \delta]$. Then

$$
\begin{aligned}
\widehat{\varphi}_{+}(c) & =\frac{\beta}{p} c^{p}|Z|_{N}-\int_{Z} F(z, c) d z \\
& \left.\leq \frac{\beta}{p} c^{p}|Z|_{N}-c_{0} c^{r}|Z|_{N} \text { (see hypothesis } \mathbf{H}(f)(v)\right) \\
& =|Z|_{N} c^{r}\left(\frac{\beta}{p} c^{p-r}-c_{0}\right)
\end{aligned}
$$

Since $p>r$, if we choose $c \in(0, \delta]$ small, then from (3.10) and (3.10) it follows that

$$
\widehat{\varphi}_{+}\left(x_{0}\right) \leq \widehat{\varphi}_{+}(c)<0
$$

hence

$$
x_{0} \neq 0 \text {. }
$$

From (3.9), we have

$$
\widehat{\varphi}_{+}^{\prime}\left(x_{0}\right)=0
$$

hence

$$
A\left(x_{0}\right)+\beta K_{p}\left(x_{0}\right)=\widehat{N}_{+}\left(x_{0}\right)
$$

where $\widehat{N}_{+}(x)():.=\widehat{f}_{+}(., x()$.$) for all x \in W_{n}^{1, p}(Z)$. On $(3.12)$, we act with the test function $-x_{0}^{-} \in W_{n}^{1, p}(Z)$ and obtain

$$
\gamma_{0}\left\|x_{0}^{-}\right\| \leq 0 \text { with } \gamma_{0}=\min \{\beta, 1\} ;
$$

hence

$$
\left.x_{0}^{-}=0 \text {, i.e., } x_{0} \geq 0, x_{0} \neq 0 \text { (see }(3.11)\right) .
$$

From (3.12) it follows that

$$
\left\{\begin{array}{l}
-\triangle_{p} x_{0}(z)+\beta x_{0}(z)^{p-1}=\widehat{f}_{+}\left(z, x_{0}(z)\right) \text { a.e. on } Z, \\
\frac{\partial x_{0}}{\partial n}=0 \text { on } \partial Z .
\end{array}\right.
$$

The nonlinear regularity theory implies that $x_{0} \in C_{+}$. Due to the sign condition (see hypothesis $\mathbf{H}(f)(v i)$ ), we have

$$
\widehat{f}_{+}\left(z, x_{0}(z)\right) \geq 0 \text { a.e. on } Z \text {. }
$$

From (3.13) and (3.14) it follows that

$$
\triangle_{p} x_{0}(z) \leq \beta x_{0}(z)^{p-1} \text { a.e. on } Z,
$$

which, by virtue of the nonlinear maximum principle of Vazquez [25], implies that

$$
x_{0} \in \operatorname{int} C_{+} .
$$

From Proposition 4 we know that $\bar{x} \in \operatorname{int} C_{+}$is a strict upper solution for problem (1.1). So, according to Definition $1(\mathrm{a})$, we have

$$
A(\bar{x})+\beta K_{p}(\bar{x})>N(\bar{x})=\widehat{N}_{+}\left(x_{0}\right) \text { in } W_{n}^{1, p}(Z)^{*},
$$

where $N(x)()=.f(., x()$.$) for all x \in W_{n}^{1, p}(Z)$. From (3.12) and (3.15) we obtain (3.16) $A(\bar{x})-A\left(x_{0}\right)+\beta\left(K_{p}(\bar{x})-K_{p}\left(x_{0}\right)\right)>\widehat{N}_{+}(\bar{x})-\widehat{N}_{+}\left(x_{0}\right)$ in $W_{n}^{1, p}(Z)^{*}$. 
On (3.16), we act with the test function $\left(x_{0}-\bar{x}\right)^{+} \in W_{n}^{1, p}(Z)$. Then, arguing as in the proof of Lemma 2, we infer that

$$
\left|\left\{x_{0}>\bar{x}\right\}\right|_{N}=0,
$$

therefore

Hence (3.13) becomes

$$
x_{0} \leq \bar{x}
$$

$$
\left\{\begin{array}{l}
-\triangle_{p} x_{0}(z)+\beta x_{0}(z)^{p-1}=f\left(z, x_{0}(z)\right) \text { a.e. on } Z \\
\frac{\partial x_{0}}{\partial n}=0 \text { on } \partial Z .
\end{array}\right.
$$

Let $0<\delta<\min _{\bar{Z}} \bar{x}$ and consider $v_{\delta}=\bar{x}-\delta \in \operatorname{int} C_{+}$. Then

$$
-\triangle_{p} v_{\delta}(z)+\beta v_{\delta}(z)^{p-1} \geq-\triangle_{p} \bar{x}(z)+\beta \bar{x}(z)^{p-1}-\sigma(\delta),
$$

with $\sigma \in C\left(\mathbb{R}_{+}\right), \sigma \geq 0$ and $\sigma(\delta) \rightarrow 0^{+}$as $\delta \rightarrow 0^{+}$. Choosing $\delta>0$ small and using (3.1), we have

$$
(\theta(z)+\varepsilon) \bar{x}(z)^{p-1}+\xi_{\varepsilon}(z)-\sigma(\delta) \geq f\left(z, x_{0}(z)\right)+\frac{\eta_{\varepsilon}}{2} \text { for a.a. } z \in Z .
$$

From $(3.6),(3.17)$ and $(3.18)$, it follows that for $\delta>0$ small, we have

$$
\begin{aligned}
h_{\delta}(z) & =-\triangle_{p} v_{\delta}(z)+\beta v_{\delta}(z)^{p-1} \\
& >f\left(z, x_{0}(z)\right)=-\triangle_{p} x_{0}(z)+\beta x_{0}(z)^{p-1} \text { a.e. on } Z .
\end{aligned}
$$

Since $h_{\delta}, f\left(., x_{0}().\right) \in L^{\infty}(Z)$, from (3.19) and Lemma 2 we infer that for $\delta>0$ small

Hence

$$
x_{0}(z) \leq v_{\delta}(z) \text { for all } z \in \bar{Z} \text {. }
$$

therefore

$$
\bar{x}(z)-x_{0}(z) \geq \delta>0 \text { for all } z \in \bar{Z},
$$

$$
\bar{x}-x_{0} \in \operatorname{int} C_{+} .
$$

Inasmuch as $x_{0} \in \operatorname{int} C_{+}$, we can find $r>0$ small such that

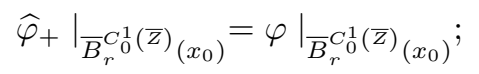

hence $x_{0} \in \operatorname{int} C_{+}$is a local $C_{n}^{1}(\bar{Z})$-minimizer of $\varphi$. Invoking Proposition 1 , we conclude that $x_{0} \in \operatorname{int} C_{+}$is a local $W_{n}^{1, p}(Z)$-minimizer of $\varphi$, and of course it solves problem (1.1).

We repeat the same process on the negative half-axis. So, because of hypotheses $\mathbf{H}(f)(i i i),(i v)$ and $(v i)$, given $\varepsilon>0$, we can find $\gamma_{\varepsilon} \in L^{\infty}(Z)_{+}, \gamma_{\varepsilon} \neq 0$ and $\widehat{\eta}_{\varepsilon}>0$ such that

$$
(\theta(z)+\varepsilon)|x|^{p-2} x-\gamma_{\varepsilon}(z) \leq f(z, x)-\widehat{\eta}_{\varepsilon} \text { for a.a. } z \in Z \text { and all } x \leq 0 .
$$

We consider the following auxiliary Neumann problem

$$
\left\{\begin{array}{l}
-\triangle_{p} v(z)+\beta|v(z)|^{p-2} v(z) \\
=(\theta(z)+\varepsilon)|v(z)|^{p-2} v(z)-\gamma_{\varepsilon}(z) \text { a.e. on } Z \\
\begin{array}{rl}
\frac{\partial v}{\partial n}=0 \text { on } \partial Z
\end{array}
\end{array}\right.
$$

Arguing as in the proof of Proposition 4, we can find $\underline{v} \in-\operatorname{int} C_{+}$, a solution of problem (3.21). By virtue of (3.20), we see that $\underline{v}$ is a strict lower solution for 
problem (1.1). Then, truncating the nonlinearity $f(z,$.$) at the points \{\underline{v}(z), 0\}$ and reasoning as in the proof of Proposition 5, we obtain:

Proposition 6. If hypotheses $\mathbf{H}(f)$ hold, then problem (1.1) admits a solution $v_{0} \in-\operatorname{int} C_{+}$which is a local minimizer of $\varphi$

Combining Propositions 5 and 6, we can summarize the results of this section in the following Theorem.

THEOREM 1. If hypotheses $\mathbf{H}(f)$ hold, then problem (1.1) admits two constant sign smooth solutions $x_{0} \in \operatorname{int} C_{+}$and $v_{0} \in-\operatorname{int} C_{+}$, which are local minimizers of the Euler functional $\varphi$.

\section{A third smooth solution}

In this section, using Morse theory, we produce a third nontrivial smooth solution for problem (1.1). Note that the Euler functional $\varphi$ satisfies the PS-condition, as one can easily verify.

In view of Theorem 1 and recalling the characterization of the critical group of a $C^{1}$-functional at a local minimizer (see Chang $[\mathbf{9}$, p. 33] and Mawhin-Willem $[\mathbf{1 8}$, p. 175]), we have:

Proposition 7. If hypotheses $\mathbf{H}(f)$ hold, then $C_{k}\left(\varphi, x_{0}\right)=C_{k}\left(\varphi, v_{0}\right)=\delta_{k, 0} \mathbb{Z}$ for all $k \geq 0$.

Next we compute the critical groups of $\varphi$ at $x=0$. Our approach is inspired by the semilinear works of Moroz $[\mathbf{1 9}]$ and Wang $[\mathbf{2 6}]$.

Proposition 8. If hypotheses $\mathbf{H}(f)$ hold, then $C_{k}(\varphi, 0)=0$ for all $k \geq 0$.

Proof. By virtue of hypotheses $\mathbf{H}(f)(i i i),(v)$ and $(v i)$, we have

$$
F(z, x) \geq c_{1}|x|^{r}-c_{2}|x|^{p} \text { for a.a. } z \in Z \text { and all } x \in \mathbb{R}
$$

with $c_{1}, c_{2}>0$. Then for $t>0$ and $x \in W_{n}^{1, p}(Z), x \neq 0$,

$$
\begin{aligned}
\varphi(t x) & =\frac{t^{p}}{p}\|D x\|_{p}^{p}+\frac{t^{p} \beta}{p}\|x\|_{p}^{p}-\int_{Z} F(z, t x(z)) d z \\
& \leq \frac{t^{p}}{p} \gamma_{1}\|x\|^{p}+t^{p} c_{2}\|x\|_{p}^{p}-t^{r} c_{1}\|x\|_{r}^{r}
\end{aligned}
$$

with $\gamma_{1}=\max \{\beta, 1\}$ (see (4.1)). Because $r<p$, from (4.2) it follows that there exists $t_{0}=t_{0}(x) \in(0,1)$ such that

$$
\varphi(t x)<0 \text { for all } t \in\left(0, t_{0}\right) .
$$

Next we show that for every $x \neq 0$

$$
\frac{d}{d t} \varphi(t x)>\frac{p}{t} \varphi(t x) \text { for all } t>0 .
$$


To this end, we remark that

$$
\begin{aligned}
\frac{d}{d t} \varphi(t x) & =\left\langle\varphi^{\prime}(t x), x\right\rangle \\
& =\langle A(t x), x\rangle+\beta t^{p-1} \int_{Z}|x|^{p} d z-\int_{Z} f(z, t x) x d z \\
& =t^{p-1}\left(\|D x\|_{p}^{p}+\beta\|x\|_{p}^{p}\right)-\frac{1}{t} \int_{Z} f(z, t x) t x d z \\
& =\frac{p}{t}\left[\frac{t^{p}}{p}\left(\|D x\|_{p}^{p}+\beta\|x\|_{p}^{p}\right)-\frac{1}{p} \int_{Z} f(z, t x) t x d z\right] \\
& >\frac{p}{t}\left[\frac{t^{p}}{p}\left(\|D x\|_{p}^{p}+\beta\|x\|_{p}^{p}\right)-\int_{Z} F(z, t x) d z\right](\operatorname{see} \mathbf{H}(f)(v i)) \\
& =\frac{p}{t} \varphi(t x),
\end{aligned}
$$

which proves (4.4).

We assume that the origin is an isolated critical point of $\varphi$, or otherwise we have a whole sequence of distinct solutions of (1.1), and so, we are done. Let $\rho>0$ be small such that $K \cap B_{\rho}=\{0\}$, where $K=\left\{x \in W_{n}^{1, p}(Z): \varphi^{\prime}(x)=0\right\}$ and $B_{\rho}=$ $\left\{x \in W_{n}^{1, p}(Z):\|x\|<\rho\right\}$. We show that for any $x \in \varphi^{0} \cap B_{\rho}$, we have $t x \in \varphi^{0} \cap B_{\rho}$ for all $t \in[0,1]$ (recall that $\varphi^{0}=\left\{x \in W_{n}^{1, p}(Z): \varphi(x) \leq 0\right\}$ ). We argue indirectly. So, suppose that for some $t_{0} \in(0,1)$, we have $\varphi\left(t_{0} x\right)>0$. Then, by continuity, there exists $t_{1} \in\left(t_{0}, 1\right]$ such that $\varphi\left(t_{1} x\right)=0$. We take $t_{1}=\min \left\{t \in\left[t_{0}, 1\right]: \varphi(t x)=0\right\}$. Hence, $\varphi(t x)>0$ for all $t \in\left[t_{0}, t_{1}\right)$ and so

$$
\left.\frac{d}{d t} \varphi(t x)\right|_{t=t_{1}} \leq 0
$$

From (4.4) and (4.5), we have

$$
0=\frac{p}{t_{1}} \varphi\left(t_{1} x\right)<\left.\frac{d}{d t} \varphi(t x)\right|_{t=t_{1}} \leq 0
$$

a contradiction. This proves that for all $x \in \varphi^{0} \cap B_{\rho}$ and all $t \in[0,1], t x \in \varphi^{0} \cap B_{\rho}$. Therefore, for every $t \in[0,1]$, the map $x \rightarrow h(t, x)=(1-t) x$ maps $\varphi^{0} \cap B_{\rho}$ into itself. Clearly, $(t, x) \rightarrow h(t, x)$ is continuous and $h(0, x)=x$ for all $x \in \varphi^{0} \cap B_{\rho}$. Hence $h$ is a continuous deformation of $\varphi^{0} \cap B_{\rho}$ to itself and so, we conclude that $\varphi^{0} \cap B_{\rho}$ is contractible into itself.

Next, we show that $\left(\varphi^{0} \cap B_{\rho}\right) \backslash\{0\}$ is contractible in itself. For this purpose, we introduce the map $T: B_{\rho} \backslash\{0\} \rightarrow(0,1]$ by

$$
T(x)=\left\{\begin{array}{lll}
1 & \text { if } & x \in\left(\varphi^{0} \cap B_{\rho}\right) \backslash\{0\} \\
t & \text { if } & x \in B_{\rho} \backslash\{0\} \text { with } \varphi(t x)=0, t \in(0,1) .
\end{array}\right.
$$

From (4.3) and (4.4) it is clear that the map $T$ is well-defined and, if $\varphi(x)>0$, then there exists a unique $T(x) \in(0,1)$ such that $\varphi(t x)<0$ for all $t \in(0, T(x))$, $\varphi(T(x) x)=0$ and $\varphi(t x)>0$ for all $t \in(T(x), 1]$. Also, we have

$$
\left.\frac{d}{d t} \varphi(t x)\right|_{t=T(x)}>\frac{p}{T(x)} \varphi(T(x) x)=0(\text { see }(4.4)) .
$$


Invoking the implicit function theorem, we infer that $x \rightarrow T(x)$ is continuous. Let $\widehat{h}: B_{\rho} \backslash\{0\} \rightarrow\left(\varphi^{0} \cap B_{\rho}\right) \backslash\{0\}$ be defined by

$$
\widehat{h}(x)=\left\{\begin{array}{lll}
T(x) x & \text { if } \quad x \in B_{\rho} \backslash\{0\}, \varphi(x) \geq 0 \\
x & \text { if } \quad x \in B_{\rho} \backslash\{0\}, \varphi(x)<0 .
\end{array}\right.
$$

The continuity of $T$ implies the continuity of $\widehat{h}$ (note that $T(x)=1$ for all $x \in$ $B_{\rho} \backslash\{0\}$ with $\left.\varphi(x)=0\right)$. Clearly $\left.\widehat{h}\right|_{\varphi^{0} \cap B_{\rho}}=\left.i d\right|_{\varphi^{0} \cap B_{\rho}}$, hence $\widehat{h}$ is a retraction and so $\left(\varphi^{0} \cap B_{\rho}\right) \backslash\{0\}$ is a retract of $B_{\rho} \backslash\{0\}$. Because $W_{n}^{1, p}(Z)$ is infinite dimensional, $B_{\rho} \backslash\{0\}$ is contractible in itself. Recall that retracts of contractible spaces are contractible too. Therefore, we infer that $\left(\varphi^{0} \cap B_{\rho}\right) \backslash\{0\}$ is contractible in itself. Consequently, from Mawhin-Willem [18, p. 172], we have

$$
C_{k}(\varphi, 0)=H_{k}\left(\varphi^{0} \cap B_{\rho},\left(\varphi^{0} \cap B_{\rho}\right) \backslash\{0\}\right) \text { for all } k \geq 0 .
$$

Next, using Proposition 2, we will compute the critical groups at infinity for the functional $\varphi$. Here we will need the restriction $p \geq 2$.

Proposition 9. If hypotheses $\mathbf{H}(f)$ hold and $2 \leq p<\infty$, then

$$
C_{k}(\varphi, \infty)=\delta_{k, 0} \mathbb{Z} \text { for all } k \geq 0 .
$$

Proof. We consider the functions

$$
(t, x) \rightarrow \varphi_{t}(x)=\frac{1}{p}\|D x\|_{p}^{p}+\frac{\beta}{p}\|x\|_{p}^{p}-(1-t) \int_{Z} F(z, x(z)) d z,
$$

for all $(t, x) \in[0,1] \times W_{n}^{1, p}(Z)$. Clearly $x \rightarrow \partial_{t} \varphi_{t}(x)$ is locally Lipschitz. Also $\varphi_{t}^{\prime}(x)=A x+\beta K_{p}(x)-t N(x)$. Since we assume $2 \leq p<\infty$, we see that $x \rightarrow \varphi_{t}^{\prime}(x)$ is locally Lipschitz too. In order to apply Proposition 2 we need to verify (2.2) and (2.3). Clearly, (2.3) holds. So, it remains to check (2.2). We proceed by contradiction. So, suppose that $(2.2)$ is not true. Then we can find sequences $\left\{t_{n}\right\}_{n \geq 1} \subseteq[0,1],\left\{x_{n}\right\}_{n \geq 1} \subseteq W_{n}^{1, p}(Z)$ such that

$$
t_{n} \rightarrow t,\left\|x_{n}\right\| \rightarrow \infty \text { and } \varphi_{t_{n}}^{\prime}\left(x_{n}\right) \rightarrow 0 \text { in } W_{n}^{1, p}(Z)^{*} .
$$

Then

$$
\left|\left\langle\varphi_{t_{n}}^{\prime}\left(x_{n}\right), u\right\rangle\right| \leq \varepsilon_{n}\|u\| \text { for all } u \in W_{n}^{1, p}(Z) \text {, with } \varepsilon_{n} \downarrow 0 \text {. }
$$

Let $y_{n}=\frac{x_{n}}{\left\|x_{n}\right\|}, n \geq 1$. By passing to a suitable subsequence if necessary, we may assume that

$$
y_{n} \stackrel{w}{\longrightarrow} y \text { in } W_{n}^{1, p}(Z) \text { and } y_{n} \rightarrow y \text { in } L^{p}(Z) .
$$

We have

$$
\begin{gathered}
\left.\left|\left\langle A\left(y_{n}\right), u\right\rangle+\beta \int_{Z}\right| y_{n}\right|^{p-2} y_{n} u d z-\left(1-t_{n}\right) \int_{Z} \frac{N\left(x_{n}\right)}{\left\|x_{n}\right\|^{p-1}} u d z \mid \\
\leq \varepsilon_{n}\|u\| \text { for all } u \in W_{n}^{1, p}(Z) .
\end{gathered}
$$

Hypotheses $\mathbf{H}(f)(i i i)$, (iv) imply that $\left\{\frac{N\left(x_{n}\right)}{\left\|x_{n}\right\|^{p-1}}\right\}_{n \geq 1} \subseteq L^{p^{\prime}}(Z)\left(\frac{1}{p}+\frac{1}{p^{\prime}}=1\right)$ is bounded. So, setting $u=y_{n}-y$ in (4.6), we have

$$
\beta \int_{Z}\left|y_{n}\right|^{p-2} y_{n}\left(y_{n}-y\right) d z \rightarrow 0 \text { and } \int_{Z} \frac{N\left(x_{n}\right)}{\left\|x_{n}\right\|^{p-1}}\left(y_{n}-y\right) d z \rightarrow 0 \text { as } n \rightarrow \infty .
$$


From (4.6) it follows that

$$
\lim _{n \rightarrow \infty}\left\langle A\left(y_{n}\right), y_{n}-y\right\rangle=0 .
$$

Invoking Proposition 3 we have that

$$
y_{n} \rightarrow y \text { in } W_{n}^{1, p}(Z),
$$

hence

$$
\|y\|=1 \text { and so } y \neq 0 .
$$

Reasoning as in the proof of Proposition 14 in Aizicovici-Papageorgiou-Staicu [2], we can show that

(4.9) $h_{n}=\frac{N\left(x_{n}\right)}{\left\|x_{n}\right\|^{p-1}} \stackrel{w}{\longrightarrow} h$ in $L^{p^{\prime}}(Z)$, with $h=g|y|^{p-2} y, g \in L^{\infty}(Z)_{+}, g \leq \theta$.

Passing to the limit as $n \rightarrow \infty$ in (4.6) and using (4.7) and (4.9), we obtain

$$
\langle A(y), u\rangle+\beta \int_{Z}|y|^{p-2} y u d z=(1-t) \int_{Z} g|y|^{p-2} y u d z .
$$

Since $u \in W_{n}^{1, p}(Z)$ is arbitrary, from (4.10) it follows that

$$
A(y)+\beta K_{p}(y)=(1-t) g K_{p}(y) .
$$

Because $t g \leq \theta$, using Lemma 1, we have

$$
\widehat{\xi}_{0}\|y\|^{p} \leq 0 \text {, hence } y=0
$$

a contradiction to (4.8). Therefore (2.2) holds for some $R>0$. Applying Proposition 2, we can say that for $c<\xi_{R}, \varphi_{0}^{c}$ is homeomorphic to a subset of $\varphi_{1}^{c}$. But note that by virtue of hypothesis $\mathbf{H}(f)(v i), \varphi_{0} \leq \varphi_{1}$, hence $\varphi_{1}^{c} \subseteq \varphi_{0}^{c}$. Therefore, $\varphi_{0}^{c}$ and $\varphi_{1}^{c}$ are homeomorphic, and so

$$
C_{k}\left(\varphi_{0}, \infty\right)=C_{k}\left(\varphi_{1}, \infty\right) \text { for all } k \geq 0
$$

Note that

$$
\varphi_{0}(x)=\varphi(x) \text { and } \varphi_{1}(x)=\frac{1}{p}\|D x\|_{p}^{p}+\frac{\beta}{p}\|x\|_{p}^{p} \text { for all } x \in W_{n}^{1, p}(Z) .
$$

Clearly, $\varphi_{1}$ has only one critical point $x=0$ and it is a global minimizer. Hence

$$
C_{k}\left(\varphi_{1}, \infty\right)=C_{k}\left(\varphi_{1}, 0\right)=\delta_{k, 0} \mathbb{Z} \text { for all } k \geq 0
$$

Since $\varphi_{0}=\varphi$, from (4.11) and (4.12), we conclude that

$$
C_{k}(\varphi, \infty)=\delta_{k, 0} \mathbb{Z} \text { for all } k \geq 0 .
$$

Now we are ready for the three solutions theorem for problem (1.1).

THEOREM 2. If hypotheses $\mathbf{H}(f)$ hold and $2 \leq p<\infty$, then problem (1.1) has at least three nontrivial smooth solutions $x_{0} \in \operatorname{int} C_{+}, v_{0} \in-\operatorname{int} C_{+}$and $y_{0} \in C_{n}^{1}(\bar{Z})$.

Proof. From Theorem 1, we already have two nontrivial smooth solutions of constant sign, namely, $x_{0} \in \operatorname{int} C_{+}$and $v_{0} \in-\operatorname{int} C_{+}$. Suppose that $0, x_{0}$ and $v_{0}$ are the only critical points of $\varphi$. Then from the Poincaré-Hopf fomula (see (2.1)) and Propositions 7, 8 and 9, we have

$$
(-1)^{0}+(-1)^{0}=(-1)^{0}
$$


hence $(-1)^{0}=0$, a contradiction. This shows that there must be a fourth critical point $y_{0} \in W_{n}^{1, p}(Z)$ of $\varphi$, distinct from $0, x_{0}$ and $v_{0}$. Evidently, $y_{0}$ is a solution of (1.1), and as before, the nonlinear regularity theory implies that $y_{0} \in C_{n}^{1}(\bar{Z})$.

REMARK 3. In fact, with some additional effort, our work can be extended to the case when in (1.1), the $p$-Laplacian is replaced by a more general operator of the form $\operatorname{div} a(z, D x(z))$, with $a(z, y)=D_{y} G(z, y)$, where $G: Z \times \mathbb{R}^{N} \rightarrow \mathbb{R}$ is measurable in $z \in Z$, of class $C^{1}$ and convex in $y \in \mathbb{R}^{N}$, and satisfies (for all $z \in Z$, $y \in \mathbb{R}^{N}$ )

$$
(a(z, y), y)_{\mathbb{R}^{N}} \leq p G(z, y) \text { and } G(z, y) \geq c\|y\|^{p} \text { for some } c>0 .
$$

Details will appear in a forthcoming paper.

\section{References}

[1] S. Aizicovici, N. S. Papageorgiou, and V. Staicu, Existence of multiple solutions with precise sign information for superlinear Neumann problems, Ann. Mat. Pura Appl., in press.

[2] S. Aizicovici, N. S. Papageorgiou and V. Staicu, Degree Theory for Operators of Monotone Type and Nonlinear Elliptic Equations with Inequality Constraints, Mem. Amer. Math. Soc. 196 (2008), no. 915.

[3] A. Ambrosetti, H. Brézis and G. Cerami, Combined effects of concave and convex nonlinearities in some elliptic problems, J. Funct. Anal. 122 (1994), 519-543.

[4] G. Anello, Existence of infinitely many weak solutions for a Neumann problem, Nonlinear Anal. 57 (2004), 199-209.

[5] T. Bartsch and S. Li, Critical point theory for asymptotically quadratic functionals and applications to problems with resonance, Nonlinear Anal. 28 (1997), 419-441.

[6] P. A. Binding, P. Drabek and Y. Huang, On Neumann boundary value problems for some quasilinear ellipic equations, Electron. J. Differential Equations 1997, no. 05, approx. 11 pp. (electronic).

[7] G. Bonanno and P. Candito, Three solutions to a Neumann problem for elliptic equations involving the p-Laplacian, Arch. Math. 80 (2003), 424-429.

[8] H. Brézis and L. Nirenberg, $H^{1}$ versus $C^{1}$ local minimizers, C. R. Acad. Sci. Paris, t. 317 (1993), 465-472.

[9] K. C. Chang, Infinite Dimensional Morse Theory and Multiple Solution Problems, Birkhäuser, Boston, 1993.

[10] F. de Paiva and E. Massa, Multiple solutions for some elliptic equations with a nonlinearity concave at the origin, Nonlinear Anal. 66(2007), 2940-2946.

[11] F. Faraci, Multiplicity results for a Neumann problem involving the p-Laplacian, J. Math. Anal. Appl. 277 (2003), 180-189.

[12] M. Filippakis, L. Gasinski and N. S. Papageorgiou, Multiplicity results for nonlinear Neumann problems, Canadian J. Math. 58 (2006), 64-92.

[13] J. Garcia Azorero, J. Manfredi and I. Peral Alonso, Sobolev versus Holder local minimizers and global multiplicity for some quasilinear elliptic equations, Commun. Contemp. Math. 2 (2000), 385-404.

[14] L. Gasinski and N. S. Papageorgiou, Nonlinear Analysis, Chapman \&Hall/ CRC Press, Boca Raton, 2006.

[15] O. Ladyzhenskaya and N. Uraltseva, Linear and Quasilinear Elliptic Equations, Academic Press, New York, 1968.

[16] S. Li, S. Wu, and H. Zhou. Solutions to semilinear elliptic problems with combined nonlinearities, J. Differential Equations 185 (2002), 200-224.

[17] G. Lieberman. Boundary regularity for solutions of degenerate elliptic equations, Nonlinear Anal. 12 (1988), 1203-1219.

[18] J. Mawhin and M. Willem, Critical Point Theory and Hamiltonian Systems, Springer-Verlag, New York, 1989.

[19] V. Moroz, Solutions of superlinear at zero elliptic equations via Morse theory, Topol. Methods Nonlinear Anal. 10 (1997), 1-11. 
[20] D. Motreanu and N. S. Papageorgiou, Existence and multiplicity of solutions for Neumann problems, J. Differential Equations 232 (2007), 1-35.

[21] K. Perera, Multiplicity results for some elliptic problems with concave nonlinearities, J. Differential Equations 140 (1997), 133-141.

[22] K. Perera and M. Schechter, Solution of nonlinear equations having asymptotic limits at zero and infinity, Calc. Var. Partial Differential Equations 12 (2001), 359-369.

[23] B. Ricceri, On three critical points theorem, Arch. Math. 75 (2000), 220-226.

[24] B. Ricceri, Infinitely many solutions of the Neumann problem for elliptic equations involving the p-Laplacian, Bull. London Math. Soc. 33 (2001), 331-340.

[25] J. Vazquez, A strong maximum principle for some quasilinear elliptic equations, Appl. Math. Optim. 12 (1984), 191-202.

[26] Z. Q. Wang, On a superlinear elliptic equation, Ann. Inst. H. Poincaré Anal. Non Linéaire 8 (1991), 43-58.

[27] S. P. Wu and H. T. Yang, A class of resonant elliptic problems with sublinear nonlinearities at origin and at infinity, Nonlinear Anal. 45 (2001), 925-935.

[28] X. Wu and K. K. Tan, On existence and multiplicity of solutions of Neumann boundary value problems for quasilinear elliptic equations, Nonlinear Anal. 65 (2006), 1334-1347.

Department of Mathematics, Ohio University, Athens, OH 45701, U.S.A.

E-mail address: aizicovi@math.ohiou.edu

Department of Mathematics, National Technical University, Zografou Campus, Athens 15780, Greece

E-mail address: npapg@math.ntua.gr

Department of Mathematics, University of Aveiro, 3810-193 Aveiro, Portugal

E-mail address: vasile@ua.pt 